\section{RESISTENCIA ANTIMICROBIANA DE Neisseria gonorrhoeae EN PERÚ}

\author{
ANTIMICROBIAL RESISTANCE OF \\ Neisseria gonorrhoeae IN PERU
Ana Jorge-Berrocal ${ }^{1 a}$, Maritza Mayta-Barrios ${ }^{\text {1b }}$, Victor Fiestas-Solórzano ${ }^{10}$

Sr Editor. La gonorrea es una infección de transmisión sexual (ITS) causada por Neisseria gonorrhoeae que se manifiesta como uretritis en los hombres y cervicitis mucopurulenta en las mujeres, con complicaciones que incluyen enfermedad inflamatoria pélvica, embarazo ectópico e infertilidad, así como una mayor transmisión del VIH y conjuntivitis gonocócica que puede producir ceguera en el recién nacido. Se estima que 78 millones de casos nuevos de infección gonocócica en personas de 15 a 49 años ocurrieron en el $2012^{(1)}$.

Según los datos del Programa Mundial de Vigilancia de Antimicrobianos para la gonorrea de la Organización Mundial de la Salud (WHO GASP, por sus siglas en ingles) entre los años 2009-2014, en el mundo se evidencia resistencia extendida a antimicrobianos como: penicilina, tetraciclina y ciprofloxacino; así como, incremento de la resistencia a azitromicina y aparición de resistencia a cefalosporinas de espectro extendido ${ }^{(2)}$.

En el Perú, un reporte de evaluación de resistencia de cepas aisladas en varias regiones durante el periodo 1998-1999 evidenciaba $100 \%$ de sensibilidad a antimicrobianos como: ciprofloxacino, espectinomicina, ceftriaxona y cefotaxima ${ }^{(3)}$. Posteriormente, el análisis de cepas aisladas en grupos de población de riesgo para ITS durante el periodo 2010-2013 evidenció $23 \%$ de resistencia a ciprofloxacino ${ }^{(4)}$.

En el marco de la vigilancia centinela de Neisseria gonorrhoeae que se implementó en 12 establecimientos de salud de siete regiones de Perú (Lima, Callao, Ancash, Ayacucho, Madre de Dios, Loreto, Ucayali), los cuales envían sus aislamientos al Instituto Nacional de Salud (Lima, Perú) para identificación bioquímica por carbohidratos en agar cistina tripteína o API®NH, y evaluación de la sensibilidad antimicrobiana mediante determinación de la concentración inhibitoria mínima por dilución en agar, utilizando los estándares del Clinical Laboratory Standards Institute (CLSI)

\footnotetext{
Instituto Nacional de Salud. Lima, Perú

a Bióloga, microbióloga; ${ }^{\text {b }}$ licenciada en Biología, magister en Microbiología Clinica; ${ }^{\mathrm{c}}$ médico infectólogo.

Recibido: 19/02/2018 Aprobado: 21/02/2018 En línea: 05/04/2018
}

Citar como: Jorge-Berrocal A, Mayta-Barrios M, Fiestas-Solórzano V. Resistencia antimicrobiana de Neisseria gonorrhoeae en Perú. Rev Peru Med Exp Salud Publica. 2018;35(1):155-6. doi: 10.17843/rpmesp.2018.351.3552. vigentes. Analizamos 96 cepas correspondientes al periodo de octubre 2016 a noviembre 2017, donde encontramos una proporción de cepas resistentes por antimicrobiano: ciprofloxacino $82,3 \%$, tetraciclina $54,2 \%$, penicilina $51 \%$, azitromicina $15,6 \%$ y por primera vez cuatro cepas con sensibilidad disminuida a ceftriaxona $(4,2 \%)$ que fueron reportadas a través del Centro Nacional de Enlace (Tabla 1).

Los fenotipos de resistencia más frecuentes fueron: CMTR/ QRNG $15,6 \%$ (resistencia cromosómica a tetraciclina/ ciprofloxacino), CMRNG/QRNG 12,5\% (resistencia cromosómica a penicilina y tetraciclina/ciprofloxacino), CMPR/QRNG 11,5\% (resistencia cromosómica a penicilina/ ciprofloxacino) y solo QRNG $10,4 \%$ (resistencia a ciprofloxacino).

De esta manera, se reporta por primera vez niveles elevados de resistencia a ciprofloxacino en Perú (82,3\%), corroborando el reporte realizado por WHO GASP (2). Esta evidencia deberá sustentar la modificación del actual esquema de tratamiento de gonorrea establecido para el país en la norma técnica vigente.

Por otro lado, aunque evidenciamos en el país un incremento de resistencia a la azitromicina $(15,6 \%)$ y por primera vez sensibilidad disminuida a ceftriaxona $(4,2 \%)$, el tratamiento doble con ceftriaxona y azitromicina (recomendado por OMS) sería el más efectivo ${ }^{(5)}$.

Sin embargo, es necesario continuar monitorizando la resistencia del gonococo a nivel nacional con la finalidad de identificar cepas que presenten menor sensibilidad a cefalosporinas de espectro extendido (actualmente la única línea de antibiótico disponible) y cuya detección debe ser notificada al Centro Nacional de Enlace por el Reglamento Sanitario Internacional ${ }^{(5)}$. En ese sentido, se debe fortalecer el sistema nacional de vigilancia (con énfasis en los casos con falla de tratamiento) y la capacidad nacional de los laboratorios, a través del aseguramiento de la calidad y realización de estudios moleculares para determinar los mecanismos de resistencia correspondientes.

Agradecimientos: Al Grupo de Vigilancia Centinela de Neisseria gonorrhoeae en el Perú: Mery Vargas-Lira del Laboratorio de Bacterias de Transmisión Sexual, Centro Nacional de Salud Pública, Instituto Nacional de Salud. Lima, Perú; Marleny TelloMinaya del Centro de Salud Tahuantinsuyo Bajo. Lima, Perú; Isabel Tarazona-Reyes del Centro de Salud Raúl Patrucco Puig. Lima, Perú, Enriqueta Dongo-Rojas del Centro de Salud Gustavo Lanatta Luján. Lima, Perú; Elsa Palomino-Balbín del Hospital Dos de Mayo. Lima, Perú; Nolberto Tangoa-Rengifo del Centro de Salud San Juan. Loreto, Perú; Carlos Peralta-Siesquen del Centro de Salud Jorge Chávez. Madre de Dios, Perú; Virginia Bedón-Izaguirre del Centro de Salud Alberto Barton. Callao, Perú; María Vilchez del Hospital La Caleta de Chimbote. Ancash, Perú; Esther Quispe-German del Centro de Salud Surquillo. Lima, Perú; Marleny Gutierrez-Rojas del Hospital Regional de Ayacucho. Ayacucho, Perú. 
Tabla 1. Susceptibilidad antimicrobiana de cepas aisladas de Neisseria gonorrhoeae en siete regiones de Perú, octubre 2016 - noviembre 2017

\begin{tabular}{|c|c|c|c|c|c|c|c|}
\hline \multirow{2}{*}{ Antibiótico } & & \multicolumn{2}{|c|}{ Resistencia } & \multicolumn{2}{|c|}{ Intermedio } & \multicolumn{2}{|c|}{ Sensible } \\
\hline & & $\mathrm{n}$ & $\%$ & $n$ & $\%$ & $\mathrm{n}$ & $\%$ \\
\hline Ciprofloxacino & $\mathrm{CIP}$ & 79 & 82,3 & 6 & 6,3 & 11 & 11,5 \\
\hline Tetraciclina & TYC & 52 & 54,2 & 33 & 34,4 & 11 & 11,5 \\
\hline Penicilina & PEN & 49 & 51,0 & 41 & 42,7 & 6 & 6,3 \\
\hline Azitromicina & AZM & 15 & 15,6 & 0 & 0,0 & 81 & 84,4 \\
\hline Ceftriaxona & CRO & 4 & 4,2 & 0 & 0,0 & 92 & 95,8 \\
\hline Espectinomicina & $\mathrm{SP}$ & 1 & 1,0 & 6 & 6,3 & 89 & 92,7 \\
\hline
\end{tabular}

Fuente: Laboratorio de Referencia Nacional de Bacterias de Transmisión Sexual, Instituto Nacional de Salud. Lima, Perú

Contribución de autoría: AJB participó en la recolección de resultados, redacción del artículo, revisión y aprobación de la versión final del manuscrito, MMB participó en el análisis e interpretación de datos, VFS revisión crítica y aprobación de la versión final del manuscrito.

Fuente de financiamiento: Instituto Nacional de Salud de Perú.

Conflictos de interés: Los autores declaran no tener conflictos de interés en la publicación del manuscrito.

\section{REFERENCIAS BIBLIOGRÁFICAS}

1. Newman L, Rowley J, Vander Hoorn S, Wijesooriya NS, Unemo $\mathrm{M}$, Low N, et al. Global Estimates of the Prevalence and Incidence of Four Curable Sexually Transmitted Infections in 2012 Based on Systematic Review and Global Reporting. PLoS One. 2015;10(12):e0143304. doi: 10.1371/journal.pone.0143304.

2. Wi T, Lahra MM, Ndowa F, Bala M, Dillon JR, Ramon-Pardo $\mathrm{P}$, et al. Antimicrobial resistance in Neisseria gonorrhoeae: Global surveillance and a call for international collaborative action. PLoS Med. 2017;14(7):e1002344. doi: 10.1371/journal. pmed.1002344.

3. Portilla J. Susceptibilidad antimicrobiana in vitro de cepas de Neisseria gonorrhoeae procesadas en el Instituto Nacional de Salud, Lima-Perú 1998-1999. Rev Peru Med Exp Salud Publica. 2003;20(4):216-9. doi: 10.17843/rpmesp.2003.204.920.

4. Tsai AY, Dueger E, Macalino GE, Montano SM, Tilley DH, Mbuchi M, et al. The U.S. military's Neisseria gonorrhoeae resistance surveillance initiatives in selected populations of five countries. MSMR. 2013;20(2):25-7.

5. Organización Panamericana de la Salud / Organización Mundial de la Salud. Alerta Epidemiológica: Neisseria gonorrhoeae con resistencia a las cefalosporinas de espectro extendido. 2 de febrero de 2018, Washington, D.C: OPS/OMS; 2018. Disponible en: https://www. paho.org/hq/index.php?option $=$ com_docman\&task $=$ doc_ view\&Itemid $=270 \&$ gid $=43500$ \&lang $=-\bar{e}$

Correspondencia: Ana Jorge Berrocal

Dirección: Psje. Trujillo 217. El Agustino, Lima

Teléfono: (+51) 7481111 anexo 2152

Correo electrónico:anae824@gmail.com

\section{ALTA RESISTENCIA ANTIMICROBIANA A FLUOROQUINOLONAS POR Campylobacter EN PACIENTES PEDIATTRICOS DE UN HOSPITAL PERUANO}

\author{
HIGH-ANTIMICROBIAL RESISTANCE TO \\ FLUOROQUINOLONES BY Campylobacter IN \\ PEDIATRIC PATIENTS IN A PERUVIAN HOSPITAL
}

\author{
Jeel Moya-Salazar ${ }^{1,2, a}$, Alfonso Terán-Vásquez ${ }^{1, b, c, d}$, \\ Richard Salazar-Hernández $z^{3, a}$
}

Sr. Editor. En el año 2017 la Organización Mundial de la Salud publicó una lista de bacterias con alta resistencia a antimicrobianos e hizo un especial llamado para la búsqueda de nuevos fármacos para luchar contra la resistencia ${ }^{(1)}$. En la lista, se incluye al Campylobacter como un patógeno de alta prioridad debido a su progresiva, alarmante y alta resistencia a fluoroquinolonas a nivel global.

Así también, en Perú se ha descrito este fenómeno de resistencia a fluoroquinolonas durante la década pasada ${ }^{(2)}$, lo cual se constituye en un riesgo y preocupación para la salud. Asimismo, se debe tener en cuenta que la campylobacteriosis es la infección bacteriana más común a nivel mundial y se presenta cada vez mayor frecuencia de resistencia a drogas de uso frecuente (macrólidos y

\footnotetext{
Servicio de Patología Clínica, Hospital Nacional Docente Madre Niño San Bartolomé. Lima, Perú.

2 Facultad de Ciencias de la Salud, Universidad Continental. Huancayo, Perú.

Hospital Nacional Guillermo Almenara Irigoyen. Lima, Perú.

Tecnólogo médico; ${ }^{\mathrm{b}}$ Licenciado en educación física; ${ }^{\mathrm{c}}$ Técnico especializado en Laboratorio clínico; ${ }^{\mathrm{d}}$ Magíster en educación.

Recibido: 08/02/2018 Aprobado: 07/03/2018 En línea: 04/04/2018
}

Citar como: Moya-Salazar J, Terán-Vásquez A, Salazar-Hernández R. Alta resistencia antimicrobiana a fluoroquinolonas por Campylobacter en pacientes pediátricos de un hospital peruano. Rev Peru Med Exp Salud Publica. 2018;35(1):156-8. doi: 10.17843/rpmesp.2018.351.3607 